\title{
STUDIES ON HEMOGLOBIN METABOLISM. I. THE KINETIC PROPERTIES OF THE PLASMA HEMOGLOBIN POOL IN NORMAL MAN *
}

\author{
BY LARS GARBY $\dagger$ AND WARD D. NOYES $\ddagger$ \\ (From the Department of Medicine, School of Medicine, University of Washington, \\ Seattle, Wash.)
}

(Submitted for publication February 9, 1959; accepted May 7, 1959)

The site of destruction of normal red cells and the subsequent fate of the liberated hemoglobin prior to its degradation is not known. Intravascular red cell rupture or phagocytosis of the red cells by the reticuloendothelial cell system or a combination of these mechanisms are the possibilities usually considered. There is some evidence to show that in some hemolytic disorders at least part of the destruction of the red cells occurs by phagocytosis. This is suggested by histological demonstration of erythrophagocytosis, the specific uptake of red cells in certain organs and the effect of splenectomy in many cases.

There are reasons to believe that the hemoglobin molecules are not reutilized to any great extent (1-4) and, since the days of Aschoff, Whipple and Mann, the reticuloendothelial cells have generally been held responsible for the breakdown of the heme moiety of the hemoglobin molecule. The chief organ site of hemoglobin breakdown is not known, although liver, spleen and bone marrow have been shown to produce bile pigments. The problem has recently been reviewed by With (5).

Hemoglobin is probably normally present in plasma in small amounts, but the turnover of the plasma hemoglobin pool is not known. Since about 1 per cent of the circulating hemoglobin mass is renewed daily, one should expect the turnover of the plasma hemoglobin to be about the same, or 6 to $7 \mathrm{Gm}$. per day, if the destruction of red cells only takes place intravascularly. The same result should be expected if the red cell

\footnotetext{
* This investigation was supported by Research Grant No. H-2994 from the United States Public Health Service. $\dagger$ In receipt of a traveling grant from the Swedish Medical Research Council. Present address: Swedish Medical Research Council Unit for Pediatric Hematology, University Hospital, Uppsala, Sweden.

$\ddagger$ United States Public Health Research Fellow of the National Heart Institute, National Institutes of Health, Bethesda, Md.
}

breakdown occurred extravascularly and the liberated hemoglobin had to be processed at sites other than those of liberation, although this latter possibility is less likely. On the other hand, if the plasma hemoglobin turnover is found to be negligible compared to 6 to $7 \mathrm{Gm}$. per day, then the possibility of intravascular breakdown cannot be seriously considered.

The present work is an attempt to define quantitatively the kinetic properties of the plasma hemoglobin pool and to evaluate the importance of this "intermediate" as a pathway in normal hemoglobin metabolism. We shall be concerned with the properties of the pool only when its hemoglobin content is below the haptoglobin binding capacity. Laurell and Nyman (6) and Jandl, Jones and Castle (7) have recently published data on the disappearance of hemoglobin from plasma in man. Their data will be discussed in the light of the present work.

\section{THEORETICAL CONSIDERATIONS}

We shall assume that the plasma hemoglobin compartment represents a pool interposed between an inflow of hemoglobin, $J_{\mathrm{in}}$, and an outflow, $J_{\text {out. }}$ The concentration of hemoglobin in plasma is $c$ and the volume of the plasma is $V$. The stationary condition is then given by:

$$
\frac{\mathrm{d} c V}{\mathrm{dt}}=J_{\text {in }}-J_{\text {out }}=0 \text {. }
$$

A stationary state obeying Equation 1 can exist for any number of values of $c$. There will also exist a number of nonstationary states for which we have:

$$
\frac{\mathrm{d} c V}{\mathrm{dt}}=J_{\text {in }}-J_{\text {out }} \neq 0 .
$$

Our purpose is then to evaluate the outflow for various values of $c$. We may reasonably suspect that the inflow, $J_{i n}$, is independent of the concentration, $c$. This will provide a complete phenomenological description of the kinetics of the pool. During the course of the analysis, a specific model will be treated. The observed experimental data are in accordance with this model. 
The simplest case, where we assume that the outflow is directly dependent on the concentration is:

$$
\frac{\mathrm{d} c V}{\mathrm{dt}}=-\mathrm{k} \cdot c+J_{\mathrm{in}}
$$

which can be ruled out on the basis of the data of Laurell and Nyman, who found an almost constant rate of elimination of injected hemoglobin between concentrations of 100 to $20 \mathrm{mg}$. per $100 \mathrm{ml}$. plasma. Jandl, Jones and Castle performed similar studies and followed the plasma concentrations down to lower values. Their data indicate a relatively constant rate of elimination at high levels, but at low levels the transfer rates decreased.

The simplest model consistent with these reported data is one in which it is assumed that the hemoglobin, during the processes responsible for its removal, enters into a series of reactions where the steady state overall reaction rate approaches a limit as $c$ increases. From measurements of the overall reaction rate in the stationary state $\left(J_{\text {out }}\right)$ and the concentration $c$, little can be learned about the details of the mechanism of the removal. However, since it will be shown that the data obtained are consistent with one rather reasonable and not too general model, this will be treated below. The hemoglobin molecules are supposed to enter into a reaction with a component or catalyst that is conserved, i.e., regenerated in some later reaction step. This first reaction may be one of physical adsorption or chemical complexing, perhaps on the surface of cells responsible for the removal. After an arbitrary number of consecutive reversible reaction steps, there follows a reaction that is practically irreversible and in which the hemoglobin is disposed of and where the catalyst is regenerated. In such a situation it can be shown (8) that the steady state overall reaction rate, in this case $J_{\text {out }}$, will depend on the concentration, $c$, in the following simple manner:

$$
J_{\text {out }}=\frac{k_{1} \cdot c}{k_{2}+c}
$$

where $k_{1}$ and $k_{2}$ are constants which in a complicated way (see Reference 8) contain the concentrations of all reactants and products, and the rate constants for their formation and degradation.

Inserting this expression for $J_{\text {out }}$ in Equation 2 we obtain :

$$
\frac{\mathrm{d} c V}{\mathrm{dt}}=J_{\mathrm{in}}-\frac{k_{1} \cdot c}{k_{2}+c}
$$

and, with the assumption that $J_{\ln }$ is independent of $c$, using Equation 1;

$$
\frac{\mathrm{d} c V}{\mathrm{dt}}=\frac{k_{1} \cdot c_{\mathrm{n}}}{k_{2}+c_{\mathrm{n}}}-\frac{k_{1} \cdot c}{k_{2}+c}
$$

where $c_{\mathrm{n}}$ is the normal concentration.

Equation 6 can be integrated to yield measured values of $c$ with respect to time in terms of the constants. If $c_{\mathrm{n}}$ and $V$ are known or measured, the constants can be evaluated by experiments in which hemoglobin is injected and its concentration determined at various time intervals. Although this approach will, in principle, be sufficient for a determination of the constants $k_{1}$ and $k_{2}$ and thus for a complete kinetic description of the system, it is clear from the integrated form of Equation 6 that the values of the constants will be critically dependent on differences between measured values of $c$ and $c_{n}$. These differences become small when the normal concentration is approached and their error can be expected to be large.

Another way of evaluating the constants is by way of a tracer experiment using labeled hemoglobin. In principle, this has the advantage of being more accurate at low concentrations. Assuming that no interfusion occurs, the tracer equation is:

$$
-c \cdot \frac{1}{c^{\prime}} \cdot \frac{\mathrm{d} c^{\prime} V}{\mathrm{dt}}=\frac{k_{1} \cdot c}{k_{2}+c}
$$

where $c^{\prime}$ is the concentration of the labeled hemoglobin; giving

$$
-\frac{\mathrm{d} \ln c^{\prime}}{\mathrm{d} t}=\frac{1}{V} \cdot \frac{k_{1}}{k_{2}+c} \text { and } \quad-\frac{\Delta \ln c^{\prime}}{\Delta t}=\frac{1}{V} \cdot \frac{k_{1}}{k_{2}+c_{\mathrm{a}}}
$$

where $c_{\mathrm{a}}$ is the average concentration during $\Delta t$. The latter integration is valid for $\Delta c^{\prime} \gg \Delta c$. In a true tracer experiment, $c$ is, of course, constant and $\operatorname{dln} c^{\prime} / \mathrm{d} t=$ constant.

It should be noted that the expression,

$$
-V \cdot c_{\mathrm{a}} \cdot \frac{\Delta \ln c^{\prime}}{\Delta t}
$$

is a measure of the flow $J_{\text {out }}$ at the particular value of $c=c_{\mathrm{a}}$ and that this measure is independent of any proposed model for the system.

Although, in general, either the nonstationary approach or the tracer approach should suffice for a complete description, it will be shown that the data are too variable to obtain the information by using one method alone. Using both approaches, however, reasonably accurate estimates of the constants can be obtained.

\section{MATERIALS AND METHODS}

Twelve healthy, adult subjects between 20 and 30 years of age, 11 male and one female, were injected intravenously with a known amount of an in vivo labeled hemoglobin solution and venous blood samples were subsequently drawn for determination of radioactivity. The experiments were performed in the morning and the subjects were not fasting. They were allowed to move freely about between samplings.

Preparation of in vivo labeled hemoglobin solution. A 32 year old white male with polycythemia vera and in good general condition was injected twice intravenously with a ferric citrate solution containing a total amount of about $0.7 \mathrm{mg}$. of iron and about $1.2 \mathrm{mc}$. of $\mathrm{Fe}^{58}$. Three to five weeks after the injection venous blood was withdrawn and hemoglobin solutions were prepared by freezing and thawing. The hemolysates were diluted and Seitz-filtered. The final solutions used for injection into the recipients contained 22 to $38 \mathrm{mg}$. per $\mathrm{ml}$. of hemoglobin and 20,000 to $30,000 \mathrm{cpm}$ per $\mathrm{ml}$. of $\mathrm{Fe}^{58}$. The amount of hemoglobin given was $1.3 \mathrm{mg}$. per $\mathrm{Kg}$. body 
weight of hemoglobin in nine subjects and $3.5 \mathrm{mg}$. per $\mathrm{Kg}$. body weight in three subjects. Aliquots of the solutions were taken and counted with the samples. The hemoglobin was determined spectrophotometrically as oxyhemoglobin by the Evelyn photoelectric colorimeter.

Estimation of plasma hemoglobin radioactivity. The plasma hemoglobin was isolated by precipitation of the proteins in acid solution, which leaves essentially all of the transferrin iron in the supernatant. To 8 to $10 \mathrm{ml}$. of plasma, $4 \mathrm{ml}$. of $2 \mathrm{~N} \mathrm{HCl}$ and $4 \mathrm{ml}$. of 20 per cent trichloracetic acid were added and the mixture stirred with a thin glass rod. The precipitate containing hemoglobin and the supernatant containing transferrin iron were separated by centrifugation. Each was wet-ashed in Kjeldahl tubes with sulphuric acid and perchloric acid. After digestion, the samples were electroplated according to the method of Peacock and associates (9). Counting was performed using a helium-filled Geiger-Müller tube. Recovery experiments revealed that about 1 per cent of added hemoglobin activity was lost to the supernatant. About 3 per cent of added radioactive transferrin was lost to the precipitate. Corrections for inefficient precipitation and coprecipitation were performed using these figures.

\section{RESULTS}

The results of the measurements of the plasma hemoglobin radioactivity as a function of time are shown in Figure 1. It is evident that the disappearance of the tracer follows relatively closely a first order reaction over the range that was observed. As the labeled hemoglobin was not injected in a

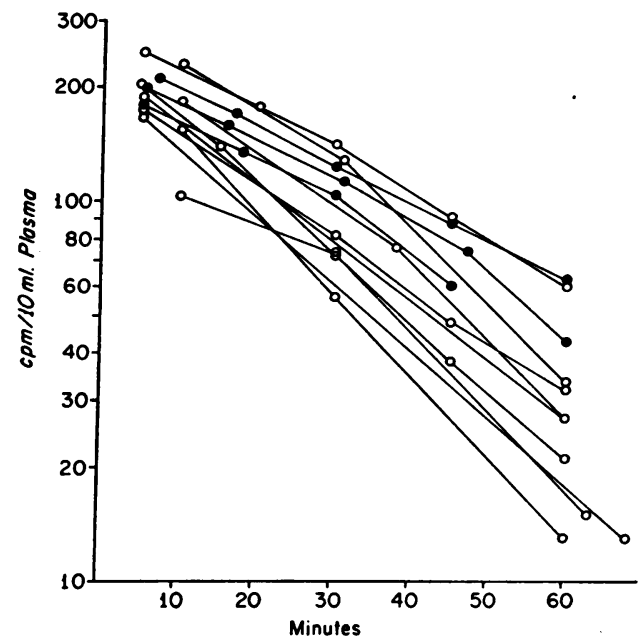

Fig. 1. The Time Course of the Radioactivity from Fe $^{50}$ Hemoglobin Injected at Zero Time is SHOWN

The dose was $1.3 \mathrm{mg}$. of hemoglobin per $\mathrm{Kg}$. body weight $(O)$ and $3.5 \mathrm{mg}$. per $\mathrm{Kg}$. body weight $(\bullet)$.

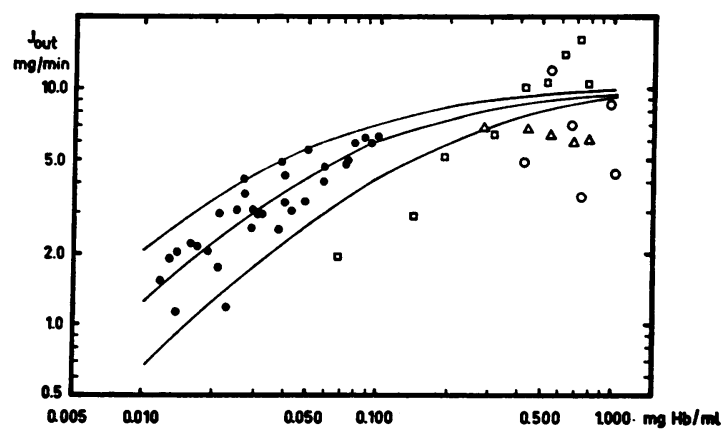

Fig. 2. The Relation Between the Flow of Hemoglobin Out of Plasma, Jout, and the Plasma HemogLobin Concentration $c$

$\mathrm{O}$ and $\triangle$ from data of Laurell and Nyman (6); from data of Jandl, Jones and Castle (7); $\bullet$, present experiments. Curves represent Equation 4 with values of constants as given in text.

true tracer dose, the slope of the disappearance curve should be slightly larger for smaller values of the hemoglobin concentration. A tendency for this can be seen in Figure 1 and is substantiated by the fact that there is a significant negative correlation (coefficient of correlation $=-0.62$ ) between the individual values of $\Delta \ln c^{\prime} / \Delta t$ and $c_{\mathrm{a}}$. The values of $c_{\mathbf{a}}$ were obtained from the number of cpm found in the plasma and the known number of cpm per mg. hemoglobin injected and a value of $0.0042 \mathrm{mg}$. per ml. added as representative for the normal hemoglobin concentration (see below).

In Figure 2 , the flow $J_{\text {out }}$ is plotted against the concentration $c$. The tracer data were obtained from Expression 9 with a plasma volume of 3,000 ml. The data of Laurell and Nyman (6) and Jandl, Jones and Castle (7) were used to give flows for average concentrations by calculating the quotient :

$\frac{c_{t_{1}}-c_{t_{2}}}{t_{2}-t_{1}}$ for $J_{\text {out }}$ and the

$$
\text { expression } \frac{c_{t_{1}}-c_{t_{2}}}{2} \text { for } c \text {. }
$$

It is evident from the figure that the relation between the outflow and the concentration is similar to one predicted by the theory. The curves are drawn by using the following values of the constants in Equation 4: upper curve $-k_{1}=$ $10, k_{2}=0.04$; middle curve $-k_{1}=10, k_{2}=$ 0.07 ; lower curve $-k_{1}=10, k_{2}=0.14$. 


\section{DISCUSSION}

The most important findings are those related to the quantitative description of the kinetics of the plasma hemoglobin pool at hemoglobin concentrations closely approaching normal. The flow rates which can be calculated from the tracer data are to a great extent independent of the validity of the details of the proposed model. However, the assumption that no interfusion occurs must be considered; i.e., that isotopic mixing between the intravascular and extravascular hemoglobin is negligible compared to the net movement of the hemoglobin. This phenomenon can be evaluated by a comparison of the disappearance kinetics of hemoglobin and albumin. Studies on the latter compound have revealed (10) that less than about 5 per cent will have left the plasma one hour after the injection. This figure is a maximum estimate for interfusion disappearance of albumin; since the haptoglobin-hemoglobin complex is considerably larger than the albumin molecule, the interfusion disappearance of the former must be even smaller. Thus, interfusion does not play a significant role in the disappearance of labeled hemoglobin from plasma and it is reasonable to assume that specific cellular mechanisms exist for the transfer.

The present data, covering plasma concentration values down to the normal range, are of particular interest in connection with the problem of the normal turnover of plasma hemoglobin. Hanks and Chaplin (11), using a modified benzidine method, have recently found the normal value of the plasma hemoglobin concentration to be 0.42 mg. per $100 \mathrm{ml}$. (range 0.25 to $0.58 \mathrm{mg}$. per 100 ml.), substantially lower than earlier estimates. If this value is accepted, the present data (Figure 2 and Equation 4 with its constants) indicate that normally only about $0.8 \mathrm{Gm}$. of hemoglobin flows in and out of the plasma compartment each day. This value corresponds to only slightly more than 10 per cent of the overall red cell hemoglobin breakdown and indicates that only a small part of the normal red cell destruction occurs intravascularly.

In a variety of hemolytic disorders Crosby and Dameshek (12) found values considerably above the normal value as accepted at that time. Provided that the capacity to remove hemoglobin from plasma is essentially normal in these cases, quite a considerable intravascular hemolysis must have occurred. Thus, in a patient with acquired hemolytic anemia, where the destruction is about six to eight times normal and the plasma hemoglobin level about $15 \mathrm{mg}$. per $100 \mathrm{ml}$., approximately twice the normal overall destruction would occur intravascularly and about three to four times the normal overall destruction would occur extravascularly. It is of considerable interest to note that the recent investigations concerning the behavior of the haptoglobin $(6,13,14)$ in various conditions are probably best interpreted in favor of increased intravascular hemolysis in hemolytic disorders.

The data presented here suggest that an adsorption process, whether the binding energy is small as in "physical" adsorption or large as in "chemical" adsorption, is intimately linked with the removal process. It should, however, be pointed out that the fact that the data are in accordance with the proposed simple model does not imply that this model necessarily exists. Several different, but physically more complicated, models might fit the data as well or better. It is, however, felt that as long as the simplest model fits the data within their accuracy, this model should be retained as a working hypothesis.

\section{SUMMARY}

The theoretical relationship between plasma hemoglobin concentration and the flow of hemoglobin out of the plasma pool has been developed for the situation in which the rate controlling step for the outflow is limited through a series of reactions involving complex formation. The tracer equation for this case has been given.

The disappearance kinetics of labeled hemoglobin from the plasma in nearly tracer amounts has been measured. The data obtained from these measurements as well as those obtained from the behavior of larger amounts of hemoglobin, as reported in the literature, are in good accordance with the proposed model. The constants for the prediction of the outflow of hemoglobin from the plasma for various concentrations of plasma hemoglobin are given.

The data indicate that the plasma hemoglobin is a relatively unimportant pathway in normal 
hemoglobin metabolism and that a few red cells die intravascularly.

\section{ACKNOWLEDGMENTS}

We wish to thank Dr. Clement A. Finch for advice and criticism and Miss Betty Loden for technical assistance.

\section{REFERENCES}

1. Sribhishaj, K., Hawkins, W. B., and Whipple, G. H. I. Bile pigment and hemoglobin interrelation in normal dogs. Amer. J. Physiol. 1931, 96, 449.

2. Hawkins, W. B., and Johnson, A. C. Bile pigment and hemoglobin interrelation in anemic dogs. Amer. J. Physiol. 1939, 126, 326.

3. Cruz, W. O., Hawkins, W. B., and Whipple, G. H. Acetylphenylhydrazine anemia. 2. Bile pigment elimination and new hemoglobin reconstruction in the bile fistula dog. Amer. J. med. Sci. 1942, 203, 848.

4. Berlin, N. I., Hewitt, C., and Lotz, C. Hippuric acid synthesis in man after the administration of $\left(\alpha \mathrm{C}^{14}\right)$ glycine. Biochem. J. 1954, 58, 498.

5. With, T. K. Biology of Bile Pigments. Copenhagen, Arne Frost-Hansen, 1954.

6. Laurell, C.-B., and Nyman, M. Studies on the serum haptoglobin level in hemoglobinemia and its influence on renal excretion of hemoglobin. Blood 1957, 12, 493.

7. Jandl, J. H., Jones, A. R., and Castle, W. B. The destruction of red cells by antibodies in man. I. Observations on the sequestration and lysis of red cells altered by immune mechanisms. J. clin. Invest. 1957, 36, 1428.

8. Langmuir, I. The adsorption of gases on plane surfaces of glass, mica and platinum. J. Amer. chem. Soc. 1918, 40, 1361.

9. Peacock, W. C., Evans, R. D., Irvine, J. W., Jr., Good, W. M., Kip, A. F., Weiss, S., and Gibson, J. G., 2nd. The use of two radioactive isotopes of iron in tracer studies of erythrocytes. J. clin. Invest. 1946, 25, 605.

10. Berson, S. A. Blood volume in health and disease. Bull. N. Y. Acad. Med. 1954, 30, 750.

11. Hanks, G. E., and Chaplin, H., Jr. Hemoglobin concentration of circulating plasma (abstract). Fed. - Proc. 1959, 18, 479.

12. Crosby, W. H., and Dameshek, W. The significance of hemoglobinemia and associated hemosiderinuria, with particular reference to various types of hemolytic anemia. J. Lab. clin. Med. 1951, 38, 829.

13. Nyman, M. Haptoglobin in pernicious anemia. Scand. J. clin. Lab. Invest. 1957, 9, 168.

14. Nosslin, B. F., and Nyman, M. Haptoglobin determination in diagnosis of haemolytic diseases. Lancet 1958, 1, 1000. 